\title{
Workshop Membership
}

\section{Attendance Dates}

H. Bojar

Onkologische Chemie

Zentrum für Physiologische Chemie

und Klinische Biochemie

Universitätsstrasse 1

D-4000 Düsseldorf

FRG(1985)

H. Boström

Department of Internal Medicine

Uppsala University

Akademisk Sjukhuser

S-7518 Uppsala

Sweden (1986)

P. Burtin

Laboratoíre d'Immunochimie

Institut de Recherches Scientifiques

sur le Cancer

Boîte Postale No. 8

F-94802 Villejuif Cedex

France $(1984,1985)$

P. Collins

Ludwig Institute for Cancer Research

Karolinska Institutet

Box 60202

S-104 01 Stockholm

Sweden (1986)

R.C. Coombes

Ludwig Institute for Cancer Research

St. George's Hospital

Tooting

GB-LondonSWMORE

UK (1985)

C.C. Cordon-Cardo

Department of Pathology

Memorial Sloan-Kettering Cancer

Center

1275 York Avenue

New York, NY 10021 
USA $(1984,1985,1986)$

H. Hirai

Tumor Laboratory Higashitokura 1-15-3 Kokunbunji-shi 185, Tokyo Japan $(1984,1985)$

D.J.R. Laurence

Institute for Cancer Research

The Haddow Laboratories

Clifton Avenue

GB-Sutton, Surrey SM2 5PX

UK(1985)

A. Malkin

Department of Clinical Biochemistry

Sunnybrook Medical Center

2075 Bayview Avenue

Toronto, Ontario M4N 3M5

Canada $(1984,1985,1986)$

A.M. Neville

(Committee Chairman)

Ludwig Institute for Cancer Research

Stadelhoferstrasse 22

CH-8001 Zurich

Switzerland

H.F. Oettgen

(Project Chairman)

Memorial Sloan-Kettering Cancer

Center

1275 York Avenue

New York, NY 10021

USA $(1984,1985,1986)$

S. Paulie

Department of Immunology The Wenner-Green Institute University of Stockholm S-106 91

Stockholm Sweden (1985)

P. Riches

Protein Reference Unit

Department of Chemical Immunology

Westminster Hospital

GB-LondonSWIP2AR

UK(1985)

C.E. Schwartz Greenwood Genetics Center 1 Gregor Mendel Circle Greenwood, SC 29646 USA

(1984, 1985, 1986)

M.K. Schwartz

Department of Clinical Chemistry

Memorial Sloan-Kettering Cancer

Center

1275 York Avenue

New York, NY 10021

USA $(1984,1985,1986)$ 
H. Shiku

Department of Immunology

Atomic Disease Institute

Nagasaki University School of Medicine

12-4 Sakamoto-cho, Nagasaki

Japan $(1985,1986)$

K. Sikora

Department of Clinical Oncology

Royal Postgraduate Medical School

Hammersmith Hospital

GB-LondonW12 0HS

UK $(1985,1986)$

U.-H. Stenman

Departments I and II of Obstetrics and

Gynecology

Helsinki University Central Hospital

SF-00290 Helsinki

Finland $(1984,1985,1986)$

H. Tate

Stone House

64 Frogges Street

Ickleton

Nr. Saffron Walden

GB-EssexCB1OlSH

UK (1985)

S. von Kleist Institut für Immunbiologie der Universität Freiburg Stefan-Meier-Strasse 8 D-7800

Freiburg i.Br. FRG(1984, 1985, 1986)

G.L. Wied

(Chairman - Cytology Working Party)

University of Chicago

1646 East 50th Street

Chicago, II 60615

USA $(1984,1985)$

Workshop Membership

61

Additional Participants and Guests
R. Arndt
G. Hundsdörfer
R. Lamerz

Abteilung fur Klinische Immunologie Gesamtprogramm für Klinikum Grosshadern Universitätskliník Hamburg Krebsbekämpfung der Universität München

Martinstrasse $52 \quad$ Koblenzstrasse 63-65 Marchioninistrasse 15
D-2000 Hamburg (FRG)
D-5300 Bonn 2 (FRG)
D-8000 München (FRG)

H. Borberg B. Konse-Thomas H.W. Schwabe

Labor für Tumorimmunologie

Deutsche Forschungsgemeinschaft Deutsche Forschungsund

Medizinische Universitätsklinik Kennedyallee $40 \quad$ Versuchsanstalt

Joseph-Stelzmannstrasse 9 D-5300 Bonn 2 (FRG) für Luft und Raumfahrt e.V. 
D-5000 Köln-Lindenthal (FRG) Linder Höhe D-5000 Köln 90 (FRG) 